\title{
Double photoemission from Ag and Pd surfaces: Energy relations
}

\author{
F. O. Schumann, ${ }^{*}$ Y. Aliaev $\odot$, I. Kostanovskiy, and J. Kirschner \\ Max Planck Institut für Mikrostrukturphysik, Weinberg 2, 06120 Halle, Germany
}

(Received 2 December 2019; revised manuscript received 28 January 2020; accepted 11 February 2020;

published 3 March 2020)

\begin{abstract}
We have investigated the electron pair emission due to single-photon absorption from $\operatorname{Ag}(100)$ and $\operatorname{Pd}(100)$ surfaces. We are interested in the energy spectra of pairs in particular near the energy cutoff. The sum energy spectra of Ag display a distinctive photon energy dependence. We also observe some fine structure. Near the high-energy cutoff the coincidence rate is too low to determine the energy position of the cutoff. Nevertheless we observe a finite signal if two $5 s p$ electrons near the Fermi level are emitted. For Pd(100) we find sum energy spectra without fine structure and the cutoff region is approached linearly. Within the experimental accuracy the minimum energy to liberate two electrons is twice the work function.
\end{abstract}

DOI: 10.1103/PhysRevB.101.115104

\section{INTRODUCTION}

As already anticipated by Einstein, a single photon can lead to the emission of more than one electron [1]. For this to occur a finite electron-electron interaction must exist [2,3]. In other words a description beyond the quasiparticle picture is mandatory. From this we have to expect that the minimum energy to eject an electron pair is different from twice the work function. This can be seen in the double ionization of the He atom. For this effect to occur an energy of $79.01 \mathrm{eV}$ is required while the single-ionization energy is $24.59 \mathrm{eV}$. We see immediately that twice this value is $49.08 \mathrm{eV}$, which is very different from the double-ionization energy. The origin of this fact is ultimately related to the electron-electron interaction.

A related effect has been observed in Auger electron spectroscopy of surfaces and has been identified with an effective electron correlation strength $U_{\text {eff }}$ [4]. For the metals Ag and Pd the reported values of $U_{\text {eff }}$ are 5.1 and $3.1 \mathrm{eV}$, respectively $[5,6]$. Specifically, these works relate to the filling of the $3 d$ core hole after photon absorption. The Auger electron emission is due to the rearrangement of the electrons from the $4 d$ valence band. More detailed information was obtained in Auger-photoelectron coincidence spectroscopy [6-8]. In this work we want to address whether a similar energy shift becomes visible in double photoemission (DPE) and investigate $\operatorname{Ag}(100)$ and $\operatorname{Pd}(100)$ surfaces at different photon energies. We expect any energy shift to be smaller than the one observed in Auger spectroscopy. This arises from the fact that the filling of the core hole will involve valence electrons in close

\footnotetext{
*schumann@mpi-halle.de
}

Published by the American Physical Society under the terms of the Creative Commons Attribution 4.0 International license. Further distribution of this work must maintain attribution to the author(s) and the published article's title, journal citation, and DOI. Open access publication funded by the Max Planck Society. proximity to the core hole. In DPE the emission involves valence electrons which can reside at different lattice sites. The characteristic distance is set by the screening length and hence the bare electron-electron interaction has to be replaced by the screened Coulomb potential. Although Pd and Ag are neighbors in the periodic table the electronic structure is very different.

In $\mathrm{Ag}$ all $4 d$ levels are occupied and lie more than $4 \mathrm{eV}$ below the Fermi level $E_{F}$. The strongly dispersing $5 s p$ band crosses the Fermi level. Due to the low occupancy of the $5 s p$ band the density of states is low at $E_{F}$. In contrast to this the $4 d$ band of Pd crosses $E_{F}$; this results in a high density of states at $E_{F}$. This means that if two electron near $E_{F}$ are emitted they will be of $5 s p$ character for $\mathrm{Ag}$ and of $4 d$ character in $\mathrm{Pd}$. Within a quasiparticle picture the strongly dispersing $5 \mathrm{sp}$ electrons can be considered to be free-electron-like and are delocalized. Consequently, we expect the electron-electron interaction to be reduced by the screening of the Coulomb interaction. This is in contrast to the $4 d$ states which are localized around the nucleus and hence the electron-electron interaction is stronger.

In the case of $\operatorname{Ag}(100)$ we observe a prominent photon energy dependence in DPE. It turns out that the self-convolution of the electronic density of states becomes a better approximation for increasing photon energy. We find some fine structure in the sum energy spectrum similar to our previous reports with lower photon energies [9]. Near the high-energy cutoff we record a finite coincidence intensity. The low coincidence intensity does not allow us to determine the exact energy position of the cutoff. Nevertheless, we can state that there is a finite probability for two $5 s p$ electrons near $E_{F}$ to be emitted.

For $\mathrm{Pd}(100)$ the coincidence intensity is higher and more detailed measurements are possible [10]. The sum energy spectra do not show a fine structure as seen for $\operatorname{Ag}(100)$. Toward the high-energy cutoff the spectra display a linear decreasing intensity very similar to our previous measurements on the Pd Auger decay [11]. Within the accuracy of this study the energy shift is zero. 


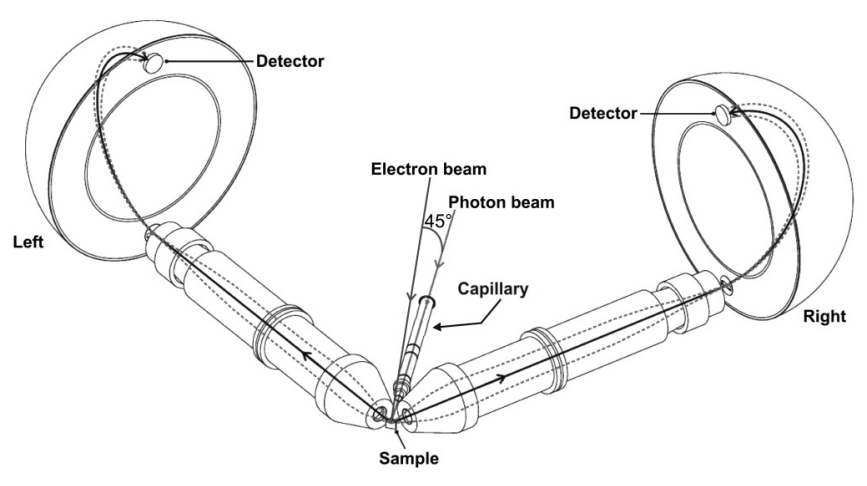

FIG. 1. The two transfer lenses of the spectrometer are symmetrically aligned with respect to the surface normal. The photon beam is aligned along the surface normal. An electron gun for $(e, 2 e)$ experiments was available; this primary beam has an angle of $45^{\circ}$ with respect to the scattering plane.

\section{EXPERIMENTAL DETAILS}

For the coincidence experiments we utilized an ultrahighvacuum chamber, which allowed the characterization via standard surface science tools like Auger spectroscopy and low energy electron diffraction. The coincidence spectrometer, sketched in Fig. 1, has been explained in more detail elsewhere [12,13].

The key components are a pair of hemispherical analyzers with $200 \mathrm{~mm}$ mean radius which we call "left" and "right," respectively. They are equipped with multichannel plate (MCP) detectors in which the impact position is determined by resistive anodes [14].

We feed the two channel plate signals and the two outputs of the resistive anodes indicating a valid impact position into a four-way logic electronics. Only if all these signals occur within a time window of $\pm 165 \mathrm{~ns}$ we will record this event. All events are saved in a list; for each event we store the impact positions on the individual MCP and the arrival time with respect to the coincidence trigger.

The substrate was either a $\operatorname{Ag}(100)$ or $\mathrm{Pd}(100)$ single crystal. Those were cleaned via $\mathrm{Ar}^{+}$sputtering and annealing. All experiments were carried out at room temperature.

We employed a standard He light source [15] for our experiments. By means of a toroidal monochromator [16] we select the He lines at 40.8 and $48.4 \mathrm{eV}$, respectively [10]. An additional measurement was performed with a photon energy of $60 \mathrm{eV}$ at the beamline UE56-2 of the synchrotron storage ring BESSY II of the HZB [17]. Coincidence measurements involving the $3 d$ levels utilized the same beamline.

\section{RESULTS}

\section{A. Energy relations}

In the DPE process, the absorption of a single photon with energy $h v$ causes the emission of two electrons. They are characterized by the binding energies $E_{v b 1}$ and $E_{v b 2}$, respectively. We adopt the notion that these energies are measured with respect to the Fermi level $E_{F}$. Therefore, the equation describing the energy balance must contain twice the work function $\phi$ and we obtain

$$
h v+E_{v b 1}+E_{v b 2}=E_{\text {left }}+E_{\text {right }}+2 \phi=E_{\text {sum }}+2 \phi .
$$

The energy sum of the emitted pair adopts its maximum if the two electrons come from the Fermi level $E_{F}$. We want to label this maximum energy sum as $E_{\text {sum }}^{\max }$. From Eq. (1) we obtain easily $E_{\text {sum }}^{\max }=h v-2 \phi$. In the last step we have assumed that the energy required to remove an electron pair is equal to twice the energy to remove a single electron. This can only be an approximation, because the electron correlation is ignored at this point as discussed in the introduction. We want to investigate whether the observed cutoff is at the same position as $E_{\text {sum }}^{\max }$. When we present sum energy curves we will plot them as a function of $E_{\text {sum }}^{\max }-E_{\text {sum. }}$. This allows the comparison of spectra obtained with different photon energies. This is the equivalent to single-electron photoemission where the emission from the $E_{F}$ is set to zero. Therefore, $E_{\text {sum }}^{\max }-E_{\text {sum }}$ is the two-particle binding energy. This entity we want to abbreviate with $E_{B}^{2 e}$.

\section{B. Signature of genuine pair emission}

Details of the coincidence setup have been given before $[13,18,19]$. It is important to recall some aspects and add additional information. For a valid coincidence event the arrival time $\left(t_{\text {left }}\right.$ and $\left.t_{\text {right }}\right)$ at the respective detector with respect to the coincidence trigger is known. This allows us to compute the arrival time histogram $d t=t_{\text {left }}-t_{\text {right }}$. The emergence of a peak is evidence of "true" coincidences as discussed in the pioneering work of Bothe and Geiger and established in the literature [20-22]. This means a pair is emitted due to impact of a single particle. In Fig. 2 we present a schematic histogram. The key difficulty is the possibility that two independent primary particles can also lead to the emission of two uncorrelated electrons termed "random" coincidences.

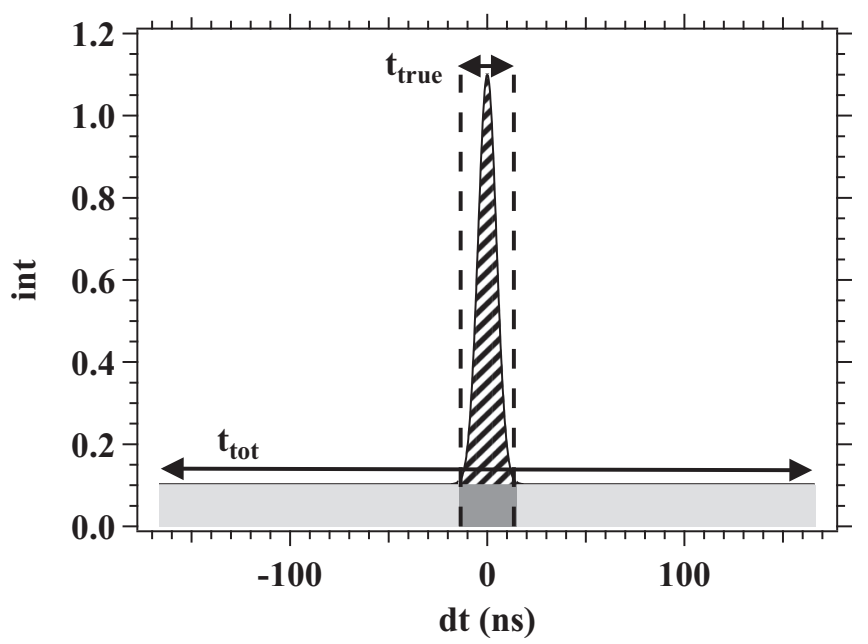

FIG. 2. Schematic arrival time histogram with a total width of $t_{\text {tot }}$. Via a Gauss fit we determine a region of interest with width $t_{\text {true }}$ as indicated by the pair of vertical dashed lines. The number of "true" events is determined by the hatched area. The light gray area determines the number of "random" events outside the peak region. The dark gray area is a measure of the "random" events in the region of interest. 
They occur for all time differences $d t$ with equal probability leading to a constant intensity outside the peak region. These unwanted events scale quadratically with the flux while the "true" coincidences scale linearly. Therefore the primary flux has to be lowered significantly until an acceptable ratio of "true" to "random" events is reached. We call this ratio in the following the TR ratio, which should be larger than 1 [23-25].

The total width of the $d t$ curve is given by $t_{\text {tot }}$ which was $330 \mathrm{~ns}$. We determine the width $t_{\text {true }}$ for true coincidences in the following way. First, we select the energy window of interest which may be the total window captured or a smaller subset. Second we perform a fit with a Gaussian curve; see Fig. 2. Third we use this result and define a region of interest where true coincidences are located. For this we take twice the full width at half maximum (FWHM) multiplied by 1.05 ; this gives the value of $t_{\text {true }}$. This is a good description of the base width of the peak indicated by the pair of vertical dashed lines; see Fig. 2. The peak width is in accordance with the time dispersion of the spectrometer [26,27]. A typical value for the FWHM is $14 \mathrm{~ns}$ for a pass energy of $150 \mathrm{eV}$ and $1 \mathrm{~mm}$ slit size for all events captured by those spectrometer settings; see Fig. 10. This width reduces to $9 \mathrm{~ns}$ if one evaluates events within a $2 \times 2 \mathrm{eV}$ window. Obviously there is a sizable contribution to the FWHM from different trajectories as discussed in the literature [26,27].

Once an energy selection has been made a $d t$ curve of this subset can be computed. The number of counts in the peak region is determined by the hatched area of the peak plus the dark gray area underneath. The latter is given by the width $t_{\text {true }}$ and the constant intensity level outside. Consequently we can state the TR ratio. The $d t$ histogram is a powerful tool, because it allows us to determine the TR ratio accurately once about 1000 counts have been recorded.

This allows us to remove the aggregate effect of the random counts in the energy spectra [24,25]. For each coincidence event we determine whether it falls within the region of interest centered around the peak of the histogram. This leads to two energy distributions; in one we have true and random counts, while the other has only random events. The window which includes random counts only has a width of $t_{\text {tot }}-t_{\text {true }}$. This we scale by a factor $t_{\text {true }} /\left(t_{\text {tot }}-t_{\text {true }}\right)$. If we take now the difference we have effectively a spectrum of true counts. There are no further adjustable parameters.

Upon approaching the energy cutoff the rate for true events will approach zero while the random rate stays roughly constant. This means the TR ratio decreases quickly below 1 and it is necessary to review carefully whether the correction is still working. Ultimately one may be forced to reduce the primary flux.

\section{Photon energy dependence Ag(100)}

We start the discussion with results obtained from a $\operatorname{Ag}(100)$ surface. We present data collected with a pass energy of $150 \mathrm{eV}$ and an entrance slit width of $3.5 \mathrm{~mm}$. This results in an energy resolution of $1.3 \mathrm{eV}$ per spectrometer. These settings were used for our material-dependent DPE study [10]. In Fig. 3 we present the 2D-energy distribution obtained with $h v=60 \mathrm{eV}$ using synchrotron radiation [17]. We have included a diagonal line which marks the maximum sum

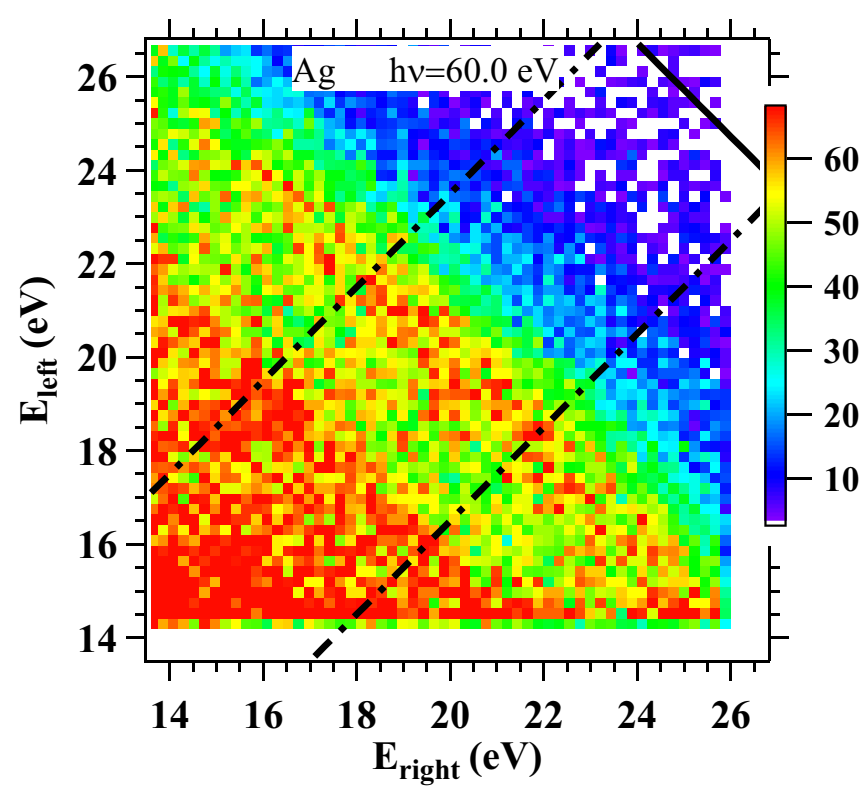

FIG. 3. The 2D-energy distribution of the "true" intensity from a DPE experiment from a $\operatorname{Ag}(100)$ surface. The photon energy was $60 \mathrm{eV}$. The solid diagonal line marks the energy position of $E_{\mathrm{sum}}^{\max }$. The constraint $\left|E_{\text {left }}-E_{\text {right }}\right| \leqslant 3 \mathrm{eV}$ is indicated by a pair of dashed lines. We will compute energy sum spectra for this selection; see Fig. 5.

energy $E_{\mathrm{sum}}^{\max }$. The pair of dashed diagonal lines indicates the region used for the generation of the sum energy spectra; see below. This region is defined by $\left|E_{\text {left }}-E_{\text {right }}\right| \leqslant 3 \mathrm{eV}$. We notice two important facts in this 2D-energy spectrum. First the intensity level near $E_{\text {sum }}^{\max }$ is low. Once the distance to the $E_{\text {sum }}^{\max }$ line increases the intensity increases, too. Second we observe a diagonal intensity ridge which is about $11 \mathrm{eV}$ below the $E_{\text {sum }}^{\max }$ line. Finally toward the lower left-hand corner the intensity rises. At this point it is useful to recall the basics of the quasiparticle band structure of Ag. The density of states (DOS) of the Ag valence band is characterized by a region of high values for the binding energy region centered at $5 \pm 2 \mathrm{eV}$ below $E_{F}$. In total there are $104 d$ electrons. The $5 s p$ band provides an almost constant contribution from about $8 \mathrm{eV}$ below up to $E_{F}$. This is much lower than those from the $4 d$ band, because the occupancy is 1 electron. From this we learn that the probability to remove two electrons from the Fermi level is strongly reduced compared to the $4 d$ band region. For the latter one requires on average $10 \mathrm{eV}$ more energy compared to two electrons from $E_{F}$. Therefore the low DPE intensity at $E_{\text {sum }}^{\max }$ and the diagonal intensity for the $h v=60 \mathrm{eV}$ experiment can be qualitatively explained.

In Fig. 4 we have defined spectral regions as $d-d$, sp- $d$, and $s p-s p$, respectively. The maximum energy of an emitted pair is attained if both electrons stem from the $s p$ band at the Fermi level. An electron from the top of the $d$ band together with an electron from $E_{F}$ has a binding energy $E_{\text {sum }}^{s p-d}$. This we identify with the lower boundary of the $s p-s p$ region and the upper bound of the $s p-d$ region. Strictly speaking the $s p-s p$ region extends to larger $E_{B}^{2 e}$ values, but the lower DOS of the $s p$ band compared to the $d$ band makes our definition a good approximation. At $E_{B}^{2 e}=8 \mathrm{eV}$ it becomes possible to eject two electrons from the top of the $d$ band, while at 


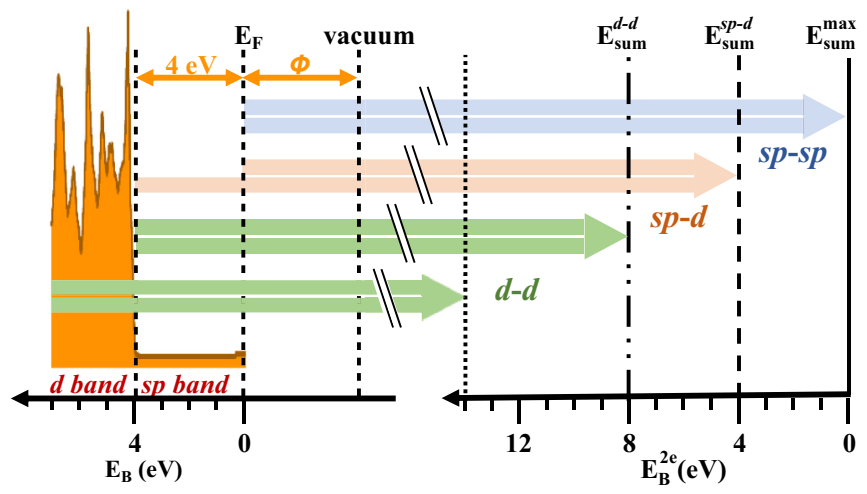

FIG. 4. Schematic picture of the DOS of Ag on the left [30]. On the right side we label spectral regions for electron pairs [9]. On the left we use the single-electron binding energy $E_{B}$, while on the right we use the two-electron binding energy $E_{B}^{2 e}$.

$E_{B}^{2 e}=14 \mathrm{eV}$ two electrons from the bottom of the $d$ band are excited. These two binding energy values determine the $d-d$ spectral region. There is an overlapping contribution from the $s p$ - $d$ part, but again the significantly higher DOS of the $d$ band compared to the $s p$ band makes our definition a good approximation.

More insight can be gained if we compute the sum energy spectrum. If we invoke the simplest picture for the DPE process we expect the sum energy spectrum to be described by the self-convolution of the DOS, termed the $2 e$ DOS in the following. This curve is included in the sum energy spectra presented in Fig. 5. We used experimental valence band spectra obtained with monochromatized $\mathrm{Al} K_{\alpha}$ radiation as DOS curves $[28,29]$. For the discussion of the $2 e$ DOS we have highlighted spectral regions with different colors. The $2 e$ DOS curve is characterized by a maximum at $E_{B}^{2 e}=11 \mathrm{eV}$. There are also kinks where the slope abruptly changes, which occurs at $E_{B}^{2 e}=7.4$ and $3.9 \mathrm{eV}$, respectively. The values define spectral regions labeled as $d-d, s p-d$, and $s p-s p$ and different colors [9]. Near $E_{B}^{2 e}=0$ the $2 e$ DOS is determined by the low DOS of the $5 s p$ electrons. At $E_{B}^{2 e}=3.9 \mathrm{eV}$ the $2 e$ DOS starts to get a contribution where one electron comes from the $4 d$ band while the other comes from the $5 s p$ band at $E_{F}$. Finally at $E_{B}^{2 e}=7.4 \mathrm{eV}$ two $4 d$ electrons become accessible.

Now we turn our attention to the $E_{\text {sum }}$ spectra measured with photon energies of 40.8, 48.4, and $60 \mathrm{eV}$. For all spectra we invoked the constraint $\left|E_{\text {left }}-E_{\text {right }}\right| \leqslant 3 \mathrm{eV}$. Although not as clear as in the $2 e$ DOS these spectra display changes in the slope. We have marked energy positions by arrows which roughly agree with the kink position of the $2 e$ DOS and are also in agreement with a recent report on DPE from a $\mathrm{Ag}(100)$ surface obtained with a much better energy resolution [9]. The arrow at $5.3 \mathrm{eV}$ in Fig. 5(a) cannot be easily understood. If we align the intensity of experiment and $2 e$-DOS curve to match at the peak then the $s p-d$ intensity is roughly a factor of 5 higher than the $2 e$ DOS for $h v=40.8 \mathrm{eV}$. This factor drops to 4 and 2 for $h v=48.4 \mathrm{eV}$ and $60 \mathrm{eV}$, respectively. While these factors are in the region as previously reported, we observe a clear photon energy dependence. This work covers a photon energy range from 40.8 to $60 \mathrm{eV}$, while the other work [9] used energies of 25.1 and $32.3 \mathrm{eV}$. Empirically

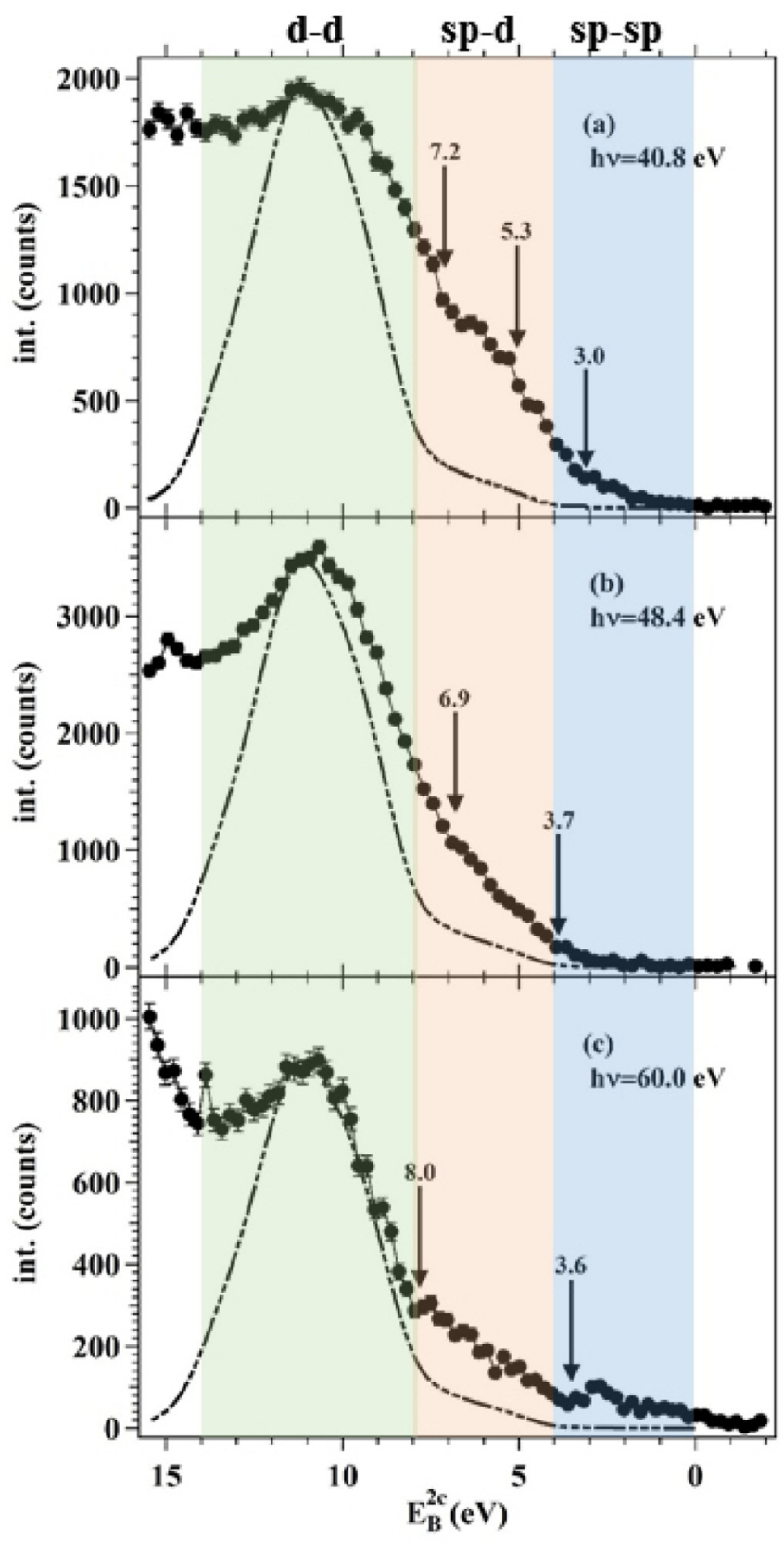

FIG. 5. The sum energy spectra for the experiment at the different photon energies is shown. The dashed curve is the $2 e$ DOS as discussed in the text. The different colored areas define spectral regions; see text.

we find that the $2 e$ DOS becomes a better approximation upon increasing the photon energy. A simple argument could be a change of the probing depth as the kinetic energy of the electrons increases. In order to explore this possibility we used the layer-resolved DOS from a $\mathrm{Ag}(100)$ sample [30]. With these data we computed the $2 e$ DOS with either the surface or bulk layer which are very similar. Therefore our data reveal a genuine photon energy dependence of the DPE process.

Care has to be taken when discussing the relative intensity levels of the highlighted spectral region in the context of the correlation strength. The enhancement of the $s p-d$ region compared to the $2 e$ DOS is obviously photon energy 


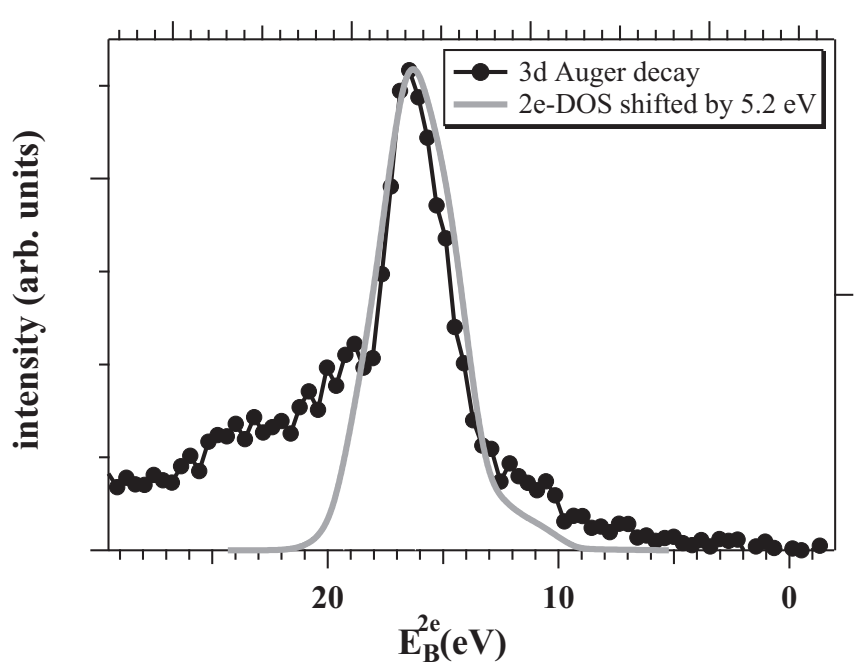

FIG. 6. We plot the $E_{\text {sum }}$ spectrum of the $3 d$ core electron and associated Auger electron from a $\operatorname{Ag}(100)$ surface [33]. The photon energy was $739 \mathrm{eV}$. We added the $2 e$ DOS from Ag and shifted it by $5.2 \mathrm{eV}$.

dependent and therefore does not only depend on the correlation strength. In related $(e, 2 e)$ studies on $\mathrm{Cu}(111), \mathrm{Cu}(100)$, and $\operatorname{Ag}(100)$ surfaces it was found that spectral regions were significantly larger than the density of states [13,31,32]. Additionally, the relative contributions of spectral ranges displayed strong primary energy dependence. This was explained within a model of electron pair diffraction along the surface normal. Such a final state effect may also be responsible for the line shape variation.

There exist Auger processes in which two electrons of the valence are missing. We pointed out in the introduction that in these cases the Auger electron energy can be significantly shifted from the expected value. In the case of $\operatorname{Ag}(100)$ we have performed coincidence measurements detecting the $3 d$ photoelectron and associated $M_{4,5} V V$ Auger electron [33]. In Fig. 6 we plot the sum energy spectrum together with the $2 e$ DOS from bulk Ag. If one shifts the $2 e$ DOS by $5.2 \mathrm{eV}$ one obtains a reasonably good agreement. This shift is a measure of the effective electron-electron interaction $U_{\text {eff }}$ which is in agreement with the literature [5,7]. Clearly the DPE measurements shown here and recently published results do not indicate an appreciable shift [9]. A key question which we want to address is the energetic position of the cutoff. The presented data so far do not allow this, because of the energy resolution. In the next section we describe an experiment with improved resolution.

\section{Behavior near the cut-off for $\operatorname{Ag}(\mathbf{1 0 0})$}

The energy spectra shown in Fig. 5 for $\mathrm{Ag}(100)$ so far have been obtained with an energy resolution of $1.3 \mathrm{eV}$. In order to address the behavior near the cutoff we reduced the size of the entrance slits from 3.5 to $1 \mathrm{~mm}$. This improves the energy resolution to $0.38 \mathrm{eV}$ at the expense of an order of magnitude smaller coincidence rate. The resulting sum energy spectrum measured with a photon energy of $48.4 \mathrm{eV}$ is shown in Fig. 7. In order to improve the statistics no constraint on the energy

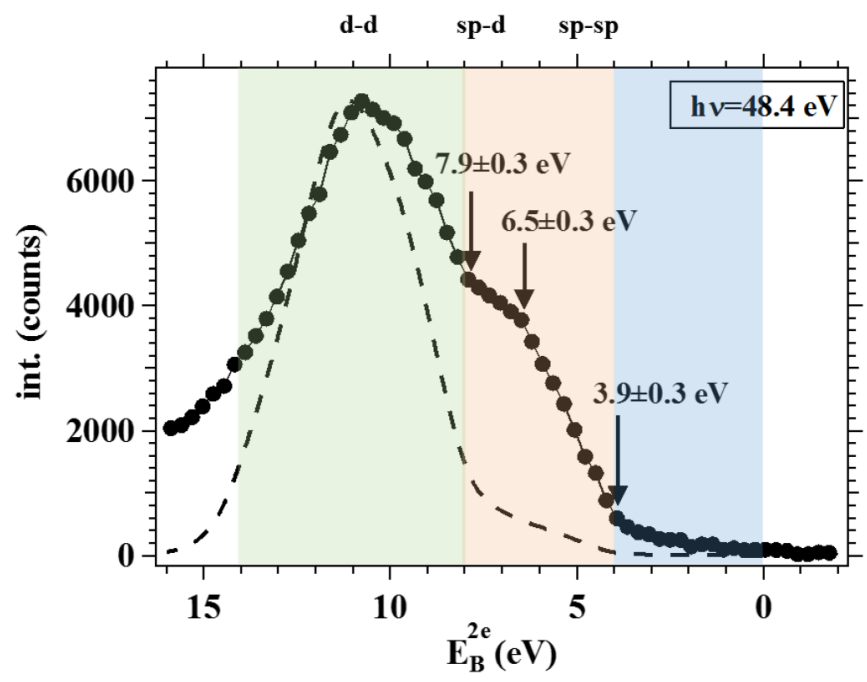

FIG. 7. Sum energy spectrum from a $\mathrm{Ag}(100)$ surface excited by $48.4 \mathrm{eV}$ photons. The dashed curve is the $2 e$ DOS for Ag. The arrows indicate energy positions where the sum energy spectrum has a change of slope. In contrast to the presentation in Fig. 5 no constraint on $E_{\text {left }}-E_{\text {right }}$ is selected.

difference $E_{\text {left }}-E_{\text {right }}$ is imposed. Again we have added as reference the $2 e$ DOS. The data presented in Fig. 7 are the sum of three individual data sets which were recorded with different primary flux and consequently different TR ratios. The acquisition time for each measurement was about 14 days. The TR ratio was 3.7, 8.1, and 13.7. For the combined set we find a ratio of 5.4. Inspection of the individual sum energy spectra showed that they possess the same shape; hence the summation is warranted. In Fig. 7 we can observe regions where the slope changes abruptly. This is much more clear than in Fig. 5(b) because of the improved energy resolution. These positions are marked by the arrows and the number is the energetic position. We do note that the arrow positions are slightly different from Fig. 5(b), because of the missing constraint on $E_{\text {left }}-E_{\text {right }}$. As discussed before the position of the arrows can be explained except for the position at $6.5 \mathrm{eV}$. These energy positions are in line with previous DPE measurements at lower photon energies [9].

Now we want to focus on the region near the cutoff. In Fig. 8 we show the dependence of the TR ratio on the sum energy. We see that the ratio drops quickly and within $4 \mathrm{eV}$ of $E_{B}^{2 e}=0$ it is below 0.5. From Fig. 7 one notes that the coincidence intensity is low in this region. These two facts highlight the experimental difficulty. As expected the signal of interest approaches zero, but at the same time the contribution of the random coincidences becomes more important. This results in TR ratios which are below 1, which are not recommended values. In principle it is easy to enhance the TR ratio by reducing the primary flux further albeit the acquisition time will be longer. This is the region where the two emitted electrons originate from the $5 s p$ band above the $4 d$ levels. We also note that the TR ratio stays finite above the cutoff value. Although the error bar increases, because of the drop of intensity there is an apparent signal. This would be in contradiction with energy conservation. We 


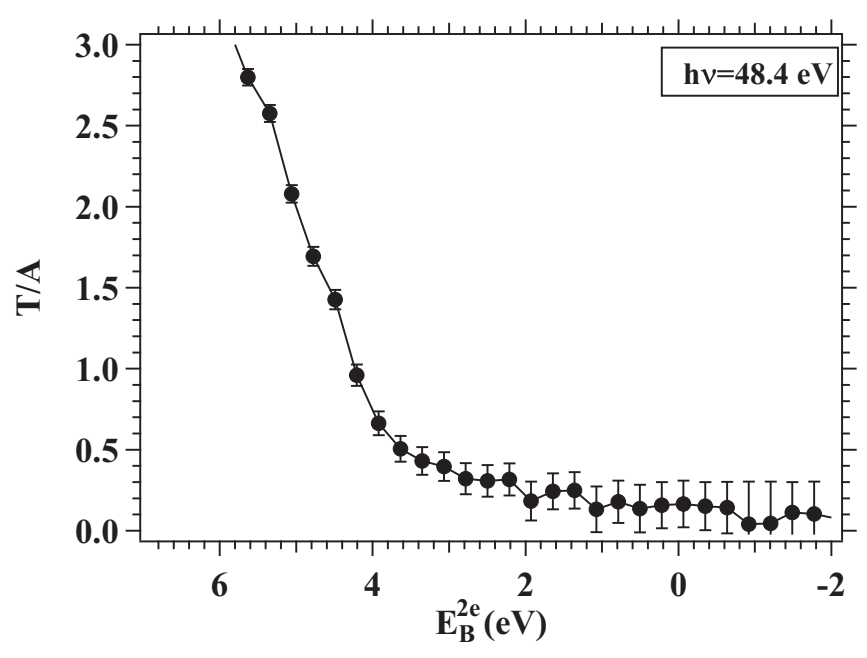

FIG. 8. Sum energy dependence of the TR ratio near the cutoff. The sample was a $\operatorname{Ag}(100)$ surface and the photon energy was $48.4 \mathrm{eV}$.

will discuss later in a separate section which effects will cause such an erroneous behavior.

Let us analyze the data in a different way. First we compute the $d t$ histogram from the combined data set. We do not use all events, but only those which are above $E_{\mathrm{sum}}^{\max }$. This histogram is shown in Fig. 9(a). This intensity is essentially constant;

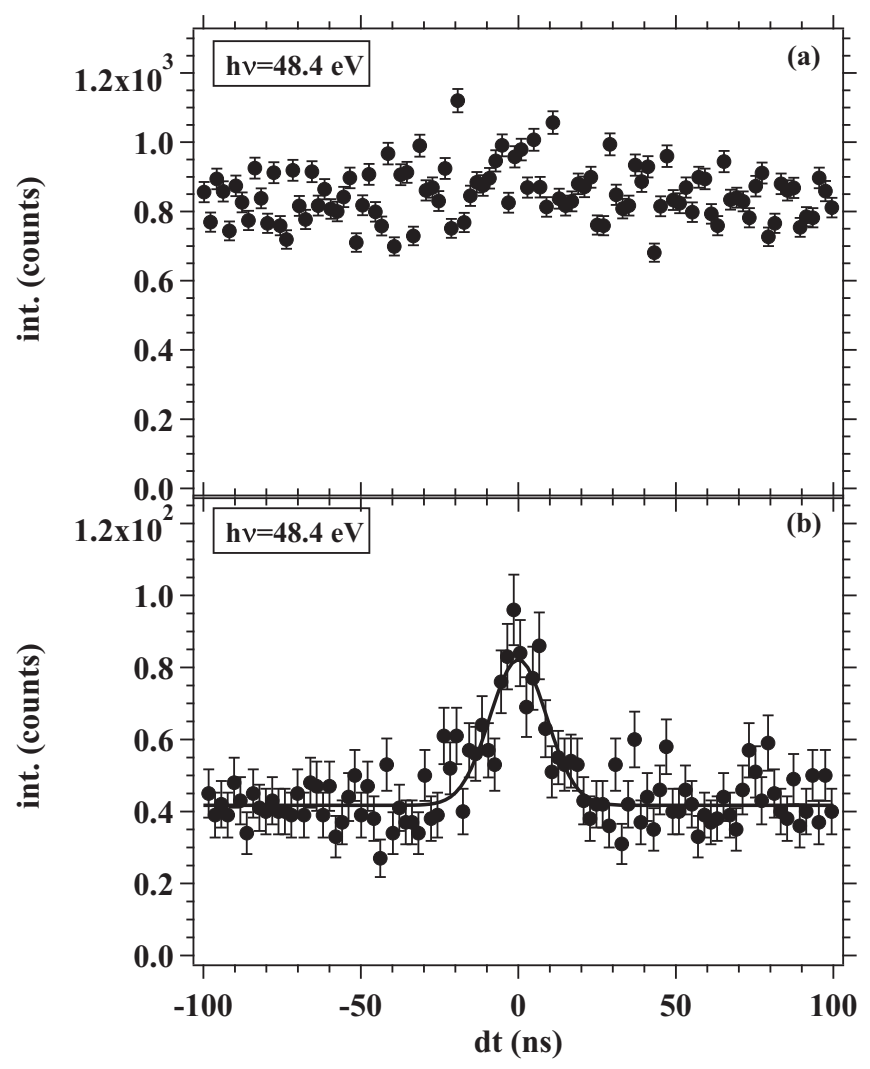

FIG. 9. In (a) we present the $d t$ histogram using all three data sets. We selected all events above $E_{\text {sum }}^{\max }$. In (b) we used the data set with the best TR ratio and selected an energy window from $E_{\text {sum }}^{\max }$ to $4 \mathrm{eV}$ below.

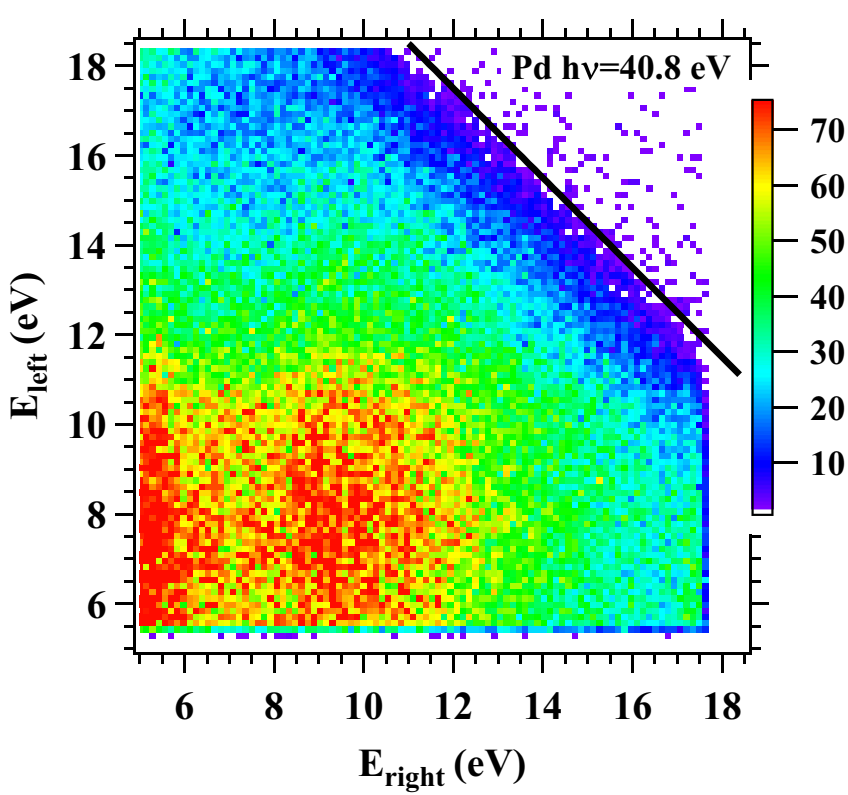

FIG. 10. We plot the 2D-energy distribution of "true" events from a $\mathrm{Pd}(100)$ surface excited by a $40.8 \mathrm{eV}$ photon beam. The diagonal line indicates the position of $E_{\mathrm{sum}}^{\max }$.

hence no evidence of true events exits in this energy range. In a second step we used the data set obtained with the best TR ratio, which was 13.4. We focus on the events in the energy window from $E_{B}^{2 e}=0$ to $4 \mathrm{eV}$. An energy window of this size prohibits an accurate determination of the cutoff position. The $d t$ histogram from this energy range is depicted in Fig. 9(b). The TR ratio of this subset is 0.5 and the coincidence rate within this energy window is $1 \times 10^{-3}$ counts $\mathrm{s}^{-1}$.

The presence of a peak proves the existence of true events. In other words the $5 s p$ electrons make a contribution to DPE. In our view the observation of true coincidences for $5 s p$ electron pairs is not a trivial result. Usually one regards these electrons as free-electron-like. One should emphasize the difference between the electron gas and free-electron gas. Both descriptions are approximations of the real interacting electron system. Common to both models is the assumption that the ionic charge is spread uniformly. As far as the electron-electron interaction is concerned it is neglected in the free-electron gas while it is incorporated in the electron gas. This interacting electron gas plays an important role in the local density approximation. A key ingredient for a finite DPE intensity is the incorporation of electron-electron interaction $[2,3]$. Crucial is the inclusion of a correlated wave function, because a quasi-single-particle picture is not sufficient.

\section{E. Energy spectra from Pd(100)}

From our previous work we have established that the DPE coincidence rate from $\mathrm{Pd}$ films is about a factor 3 higher than for the $\operatorname{Ag}(100)$ surface [10]. Additionally we expect a higher DPE rate near $E_{\text {sum }}^{\max }$ due to higher DOS of the $4 d$ states near $E_{F}$. This is in contrast to $\mathrm{Ag}$ in which case the region near $E_{F}$ is determined by $5 s p$ electrons with a lower DOS. In Fig. 10 we show the 2D-energy spectrum from a Pd(100) surface excited by $40.8 \mathrm{eV}$ photons. This distribution 
is not symmetric with respect to the line $E_{\text {left }}=E_{\text {right }}$. This is a consequence of the difficulty to achieve perfect alignment between the two spectrometers and the light source. There are no obvious features visible as in the case for $\operatorname{Ag}(100)$ excited with $60 \mathrm{eV}$; see Fig. 3. Toward the $E_{\text {sum }}^{\max }$ line the intensity drops smoothly.

The sum energy spectra for photon energies of 40.8 and $48.4 \mathrm{eV}$ are plotted in Fig. 11 without any constraints on $E_{\text {left }}-E_{\text {right }}$. In panels (a) and (b) we present almost the
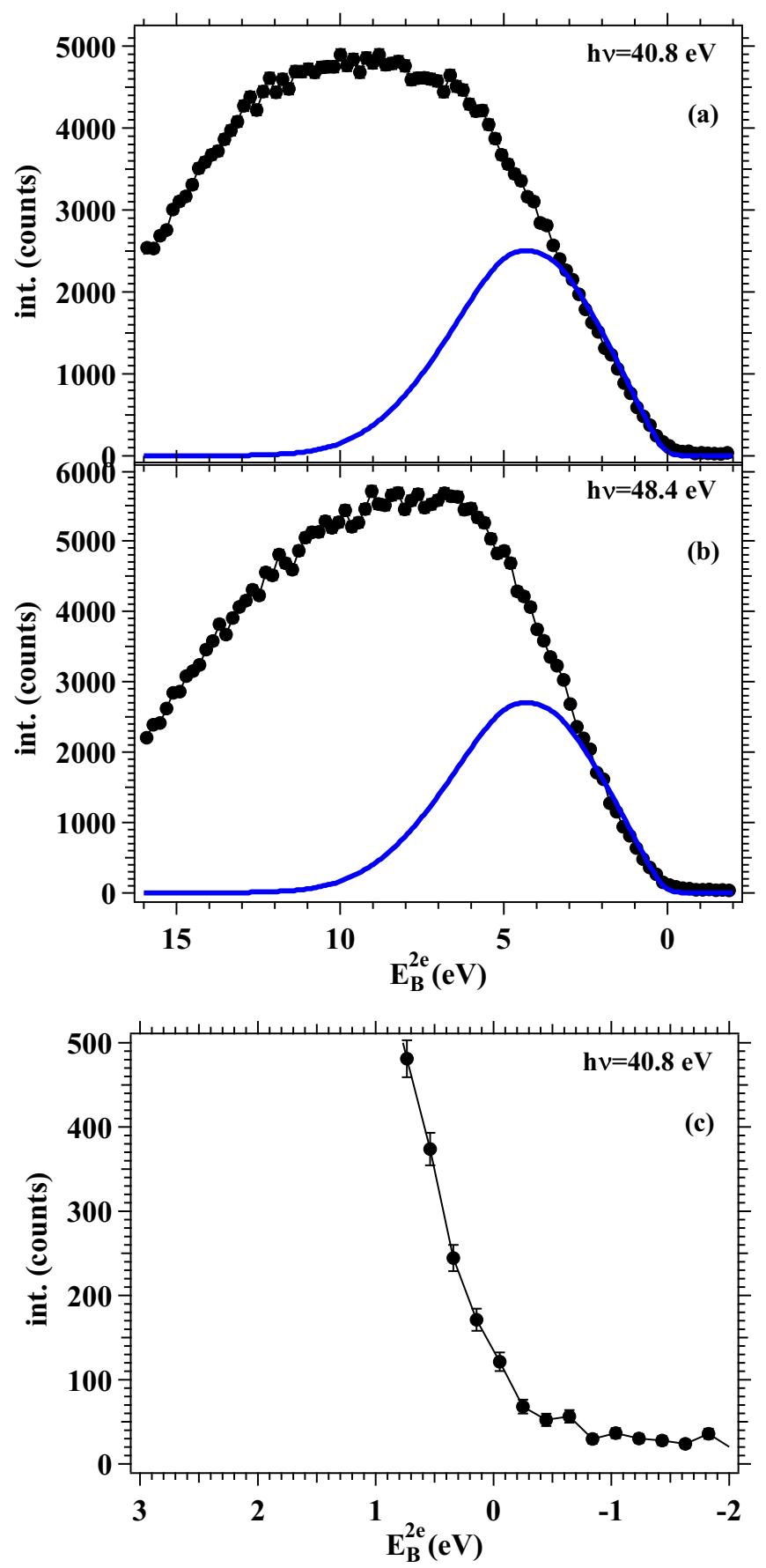

FIG. 11. Panels (a) and (b) display the sum energy spectra from a $\operatorname{Pd}(100)$ surface with a photon energy of 40.8 and $48.4 \mathrm{eV}$, respectively. In both panels we added the $2 e$ DOS of $\mathrm{Pd}$ as a blue curve. In (c) we zoom into the region near $E_{B}^{2 e}=0$ for $40.8 \mathrm{eV}$ photon energy. complete spectra while in (c) we zoom into the region near $E_{B}^{m 2 e}=0$ for $h v=40.8 \mathrm{eV}$. We have added to panels (a) and (b) the $2 e$ DOS of Pd as a blue curve. Analogously to the $\mathrm{Ag}$ case we used experimental valence band spectra as the DOS for self-convolution [29]. We have scaled the $2 e$ DOS such that it aligns best with the experimental data near the cutoff. We see that both curves display a linear decrease toward the cutoff. Experimentally we observe that the linear region covers a range of about $6 \mathrm{eV}$. While the linear region between $E_{B}^{2 e}=4 \mathrm{eV}$ and $0 \mathrm{eV}$ can be reproduced the further increase of the intensity for $E_{B}^{2 e}>4 \mathrm{eV}$ cannot be explained by the $2 e$ DOS. As a matter of fact the $2 e$ DOS suggests an almost vanishing intensity, while the experiment shows the highest intensity for $E_{B}^{2 e}=10 \mathrm{eV}$. The $E_{\text {sum }}$ spectrum for a photon energy of $48.4 \mathrm{eV}$ is presented in Fig. 11(b). It is very similar to the result obtained with $h v=40.8 \mathrm{eV}$. The main difference is the behavior for $E_{B}^{2 e}$ below $10 \mathrm{eV}$ where the intensity for $h v=48.4 \mathrm{eV}$ starts to decrease, whereas for $h v=40.8 \mathrm{eV}$ it still is close to the maximum. In Fig. 11(c) we zoom into the region near $E_{B}^{2 e}=0$. As stated above we observe intensity up to $2 \mathrm{eV}$ above $E_{\text {sum }}^{\max }$. The origin of this intensity will be discussed below.

The higher coincidence rate of $\mathrm{Pd}(100)$ compared to $\operatorname{Ag}(100)$ allowed us to operate with improved spectrometer resolution. We reduced the pass energy from 150 to $50 \mathrm{eV}$, while the slit size was kept constant. Therefore the resolution per spectrometer changed from $375 \mathrm{meV}$ to $125 \mathrm{meV}$. This results in an energy resolution of $180 \mathrm{meV}$ in the sum energy. As a consequence the energy window captured by each spectrometer was reduced by a factor of 3 which amounts to $4.5 \mathrm{eV}$. This change in the spectrometer setting caused a dramatic reduction of the coincidence rate from 1.2 to 0.025 counts $\mathrm{s}^{-1}$. In Fig. 12 we show the behavior of the sum energy spectrum near $E_{\text {sum }}^{\max }$ without any constraint on

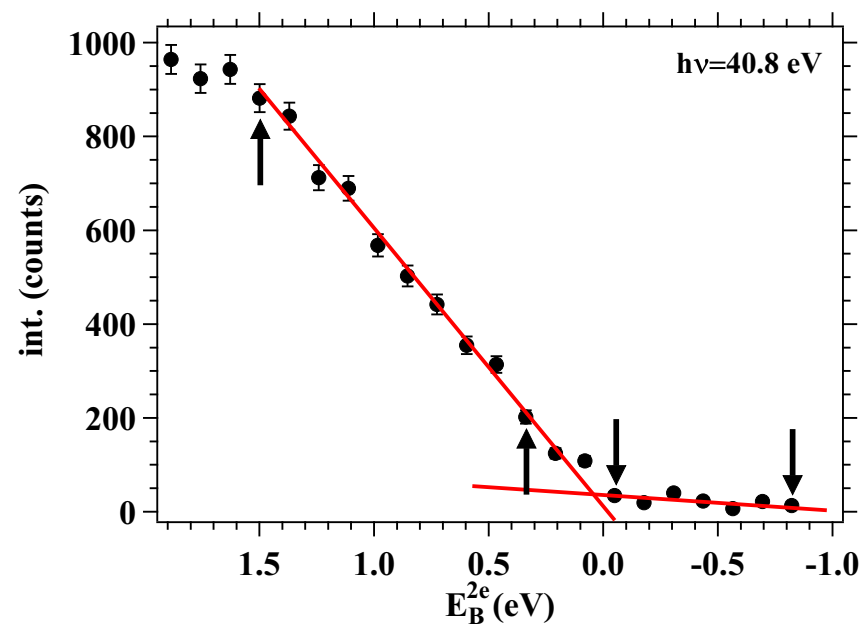

FIG. 12. We plot the sum energy spectrum from a Pd(100) surface excited by $40.8 \mathrm{eV}$ photons. Compared to the data in Fig. 11 the energy resolution is 3 times better. We focus on the region near $E_{\text {sum }}^{\max }$. The red lines are linear extrapolations from the regions above and below $E_{B}^{2 e}=0$. The two upward (downward) arrows indicate the range used for the linear fit below (above) the cutoff. They intercept $0.05 \mathrm{eV}$ below $E_{B}^{2 e}=0$. 
$E_{\text {left }}-E_{\text {right }}$. We see that the intensity decreases linearly upon approaching this point in agreement with Fig. 11. There is also a tailing of intensity toward higher energies. Both regimes can be approximated by linear curves, which are added to Fig. 12. The energy position of the intercept of these red curves can be identified as the cutoff value. Within the experimental accuracy it is at $E_{B}^{2 e}=0 \mathrm{eV}$; this means we observe no apparent shift. We are not aware of a theoretical prediction on the energy shift. Clearly the potential shift is significantly smaller than the related shift of the $\mathrm{Pd} 3 d$-Auger decay. For this transition a value of $U_{\text {eff }}=3.2 \mathrm{eV}$ is reported [6].

\section{ON SYSTEMATIC ERRORS IN ENERGY SPECTRA}

We have pointed out that our procedure to remove the aggregate effect of the random coincidences yields intensity above the expected cutoff value. This would defeat energy conservation, because we expect a potential shift toward lower energies due to electron correlation. In the course of this study we recognized two experimental issues which can give intensity at higher energies.

The He light source emits intensity for a few narrow lines. The monochromator allows the selection of a desired line, while the others are suppressed. This will not be perfect as reported in the literature for a monochromator model identical to our device [34]. The question arises as to whether it is possible that besides the selected $40.8 \mathrm{eV}$ line also some contribution from the lines at 48.4 and $51.0 \mathrm{eV}$ is present. We performed a noncoincidence experiment in which one spectrometer covered an energy window for single photoemission with $40.8 \mathrm{eV}$. The other spectrometer could only detect the photoemission intensity from the $48.4 \mathrm{eV}$ line. We found that the count rate for the $40.8 \mathrm{eV}$ line was about a factor 500 higher than for the $48.4 \mathrm{eV}$ line. We may take this as measure of the photon flux and expect a replica of the sum energy spectrum for $40.8 \mathrm{eV}$. The replica intensity is about a factor of 500 smaller but shifted by $7.6 \mathrm{eV}$ toward higher energies. This contribution does not exist if a primary electron gun is used for pair emission. We performed additional $(e, 2 e)$ experiments on the $\operatorname{Pd}(100)$ surface and found that the tailing of true events above $E_{\text {sum }}^{\max }$ exists there, too. For such a measurement we kept the spectrometer settings fixed and adjusted the primary electron energy to put the $E_{\text {sum }}^{\max }$ line to be at the same position as for the DPE experiment. This shows that there must be another issue unrelated to an incomplete suppression of photon energies higher than the selected line.

The impact position of an electron on the MCP is determined by a resistive anode [14]. Test measurements of the manufacturer showed that a narrow electron beam results in a FWHM of $1 \%$ of the diameter of the MCP. It turns out that for operation of slit sizes of $1 \mathrm{~mm}$ or larger the resulting energy uncertainty can be ignored. Under these conditions the energy resolution is determined by the slit size. For example, a pass energy of $150 \mathrm{eV}$ and $1 \mathrm{~mm}$ slits determines the spectrometer resolution to be $0.38 \mathrm{eV}$. The position error translates to an energy uncertainty of $0.1 \mathrm{eV}$. However, a narrow electron beam striking the MCP will also have a small intensity outside the peak region. For a pass energy of $150 \mathrm{eV}$ this region extends up to $0.8 \mathrm{eV}$ above or below the peak position. We conclude that incomplete suppression of He lines and the spatial resolution of the MCP will cause true coincidences to appear above the cutoff value.

\section{SUMMARY}

We have discussed the DPE energy spectra of $\operatorname{Ag}(100)$ and $\operatorname{Pd}(100)$ surfaces. We observe a photon energy dependence for the $\operatorname{Ag}(100)$ surface if the photon energy is varied between 40.8 and $60 \mathrm{eV}$. The relative contribution of $s p$ - $d$ pairs compared to $d$ - $d$ pairs decreases as a function of photon energy. We have evidence of true coincidences in the vicinity of $E_{\mathrm{sum}}^{\max }$ which stem from the $5 s p$ states. The low coincidence rate prohibits a determination of the cutoff value.

The energy spectra from a $\operatorname{Pd}(100)$ crystal do not exhibit any fine structure. As a matter of fact over a range of about $6 \mathrm{eV}$ the intensity decreases linearly toward the cutoff value. The minimum energy to eject two electrons from a $\operatorname{Pd}(100)$ surface is within experimental accuracy twice the work function.

\section{ACKNOWLEDGMENTS}

We thank the HZB for the allocation of synchrotron beamtime at the BESSY storage ring. This work was funded by the Deutsche Forschungsgemeinschaft (DFG, German Research Foundation), Project No. 31047526 and SFB 762, Project No. B7.
[1] A. Einstein, Ann. Phys. 322, 132 (1905).

[2] J. L. Powell and B. Crasemann, Quantum Mechanics (AddisonWesley, Reading, MA, 1961).

[3] J. Berakdar, Phys. Rev. B 58, 9808 (1998).

[4] E. Antonides, E. C. Janse, and G. A. Sawatzky, Phys. Rev. B 15, 1669 (1977).

[5] A. C. Parry-Jones, P. Weightman, and P. T. Andrews, J. Phys. C 12, 1587 (1979).

[6] M. T. Butterfield, R. A. Bartynski, and S. L. Hulbert, Phys. Rev. B 66, 115115 (2002).

[7] D. A. Arena, R. A. Bartynski, R. A. Nayak, A. H. Weiss, and S. L. Hulbert, Phys. Rev. B 63, 155102 (2001).
[8] D. A. Arena, R. A. Bartynski, R. A. Nayak, A. H. Weiss, S. L. Hulbert, and M. Weinert, Phys. Rev. Lett. 91, 176403 (2003).

[9] A. Trützschler, M. Huth, C.-T. Chiang, R. Kamrla, F. O. Schumann, J. Kirschner, and W. Widdra, Phys. Rev. Lett. 118, 136401 (2017).

[10] F. O. Schumann, Y. Aliaev, I. Kostanovskiy, G. Di Filippo, Z. Wei, and J. Kirschner, Phys. Rev. B 93, 235128 (2016).

[11] I. Kostanovskiy, F. O. Schumann, Y. Aliaev, Z. Wei, and J. Kirschner, J. Phys.: Condens. Matter 28, 015601 (2016).

[12] G. A. van Riessen, F. O. Schumann, M. Birke, C. Winkler, and J. Kirschner, J. Phys.: Condens. Matter 20, 442001 (2008). 
[13] F. O. Schumann, R. S. Dhaka, G. A. van Riessen, Z. Wei, and J. Kirschner, Phys. Rev. B 84, 125106 (2011).

[14] Quantar Technology Incorporated, model 3300, series MCP, http://www.quantar.com/pages/QTI/ofs.htm.

[15] Light source MBS L-1 from MB Scientific, www.mbscientific. se/products.php?ca $=30 \& \mathrm{pr}=61 \& \mathrm{~m}=52$.

[16] Monochromator VUV5040 from VG Scienta, https://www. scientaomicron.com/en/productDetailPages/Components/ photon-sources/VUV5k.

[17] K. J. S. Sawhney, F. Senf, M. Scheer, F. Schäfers, J. Bahrdt, A. Gaupp, and W. Gudat, Nucl. Instrum. Methods A 390, 395 (1997).

[18] G. A. van Riessen, F. O. Schumann, M. Birke, C. Winkler, and J. Kirschner, J. Phys.: Conf. Ser. 185, 012051 (2009).

[19] G. A. van Riessen, Z. Wei, R. S. Dhaka, C. Winkler, F. O. Schumann, and J. Kirschner, J. Phys.: Condens. Matter 22, 092201 (2010).

[20] W. Bothe and H. Geiger, Z. Phys. A 32, 639 (1925).

[21] I. E. McCarthy and E. Weigold, Phys. Rep. 27, 275 (1976).

[22] S. Thurgate, Surf. Interface Anal. 20, 627 (1993).

[23] H. W. Haak, G. A. Sawatzky, L. Ungier, J. K. Gimzewski, and T. D. Thomas, Rev. Sci. Instrum. 55, 696 (1984).
[24] G. A. Sawatzky, Auger photoelectron coincidence spectroscopy, in Auger Electron Spectroscopy, edited by C. L. Briant and R. P. Messmer (Academic Press, San Diego, 1988).

[25] E. Jensen, R. A. Bartynski, S. L. Hulbert, and E. Johnson, Rev. Sci. Instrum. 63, 3013 (1992).

[26] M. Völkel and W. Sandner, J. Phys. E 16, 456 (1983).

[27] O. Kugeler, S. Marburger, and U. Hergenhahn, Rev. Sci. Instrum. 74, 3955 (2003).

[28] S. Hüfner, G. K. Wertheim, N. V. Smith, and M. M. Traum, Solid State Commun. 11, 323 (1972).

[29] S. Hüfner, G. K. Wertheim, and D. N. E. Buchanan, Solid State Commun. 14, 1173 (1974).

[30] L. Pasquali, M. C. Sapet, E. M. Staicu-Casagrande, P. Cortona, V. A. Esaulov, S. Nannarone, M. Canepa, S. Terreni, and L. Mattera, Nucl. Instrum. Methods B 212, 274 (2003).

[31] Z. Wei, F. O. Schumann, R. S. Dhaka, and J. Kirschner, Phys. Rev. B 85, 195120 (2012).

[32] F. O. Schumann, L. Behnke, C. H. Li, J. Kirschner, Y. Pavlyukh, and J. Berakdar, Phys. Rev. B 86, 035131 (2012).

[33] Z. Wei, F. O. Schumann, C. H. Li, L. Behnke, G. Di Filippo, G. Stefani, and J. Kirschner, Phys. Rev. Lett. 113, 267603 (2014).

[34] T. Greber, O. Raetzo, T. J. Kreutz, P. Schwaller, W. Deichmann, E. Wetli, and J. Osterwalder, Rev. Sci. Instrum. 68, 4549 (1997). 